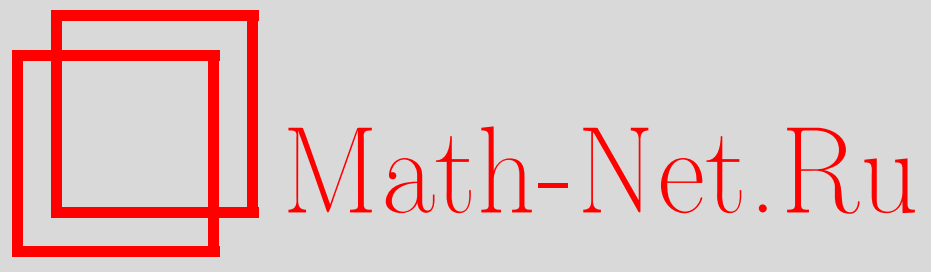

А. В. Цыганов, О бигамильтоновых системах натурального вида, ТМФ, 2006, том 149, номер 2, 161-182

DOI: https://doi.org/10.4213/tmf4225

Использование Общероссийского математического портала Math-Net.Ru подразумевает, что вы прочитали и согласны с пользовательским соглашением http://www . mathnet.ru/rus/agreement

Параметры загрузки:

IP: 54.162 .27 .143

26 апреля 2023 г., 13:04:15

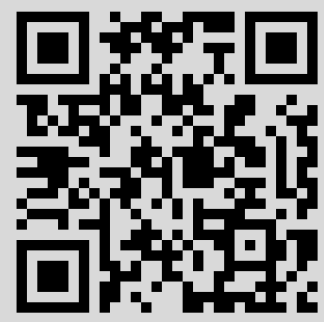




\title{
2006 г.
}

\author{
А. В. Цыганов*
}

\section{О БИГАМИЛЬТОНОВЫХ СИСТЕМАХ НАТУРАЛЬНОГО ВИДА}

\begin{abstract}
Предложен новый метод построения интегрируемых систем натурального вида, в котором интегралы движения являются решениями переопределенной системы алгебраических и дифференциальных уравнений в частных производных, полученной из условия совместности тензоров Пуассона, полиномиальных по импульсам, и условия инвариантности соответствующего интегралам движения билагранжева распределения относительно действия оператора рекурсии.
\end{abstract}

Ключевые слова: интегрируемые системы, бигамильтоновы многообразия, разделение переменных.

\section{1. ВВЕДЕНИЕ}

Гладкое многообразие $M$, снабженное парой совместных тензоров Пуассона $P$ и $P^{\prime}$, называется бигамильтоновым многообразием. Динамические системы на многообразии $M$, обладающие достаточным для интегрируемости по Лиувиллю количеством функционально независимых интегралов движения $H_{1}, H_{2}, \ldots, H_{n}$ в биинволюции относительно скобок Пуассона

$$
\left\{H_{i}, H_{k}\right\}=\left\{H_{i}, H_{k}\right\}^{\prime}=0,
$$

будем называть для краткости биинтегрируемыми системами или обобщенными бигамильтоновыми системами [1].

Наиболее общим методом поиска интегрируемых систем, который применим практически ко всем известным системам, является метод неопределенных коэффициентов [2], при этом интегралы движения $H_{i}$ ищутся в виде полиномов или других функций с неопределенными коэффициентами, которые затем находятся из условия инволютивности интегралов движения (1.1).

В данной работе рассмотрена модификация метода неопределенных коэффициентов, которая может быть использована для поиска тензоров Пуассона $P^{\prime}$ и связанных с ними бигамильтоновых систем натурального вида. В отличие от исходного метода, предлагаемый алгоритм позволяет получить не только интегралы движения, но и оператор рекурсии вместе с соответствующими ему переменными разделения.

* Санкт-Петербургский государственный университет, Санкт-Петербург, Россия. E-mail: tsiganov@mph.phys.spbu.ru 
В качестве примера построены операторы рекурсии для известных интегрируемых систем натурального вида с интегралами движения третьей и четвертой степени по импульсам.

\section{2. ИНТЕГРИРУЕМЫЕ СИСТЕМЫ НАТУРАЛЬНОГО ВИДА НА $\omega N$-МНОГООБРАЗИЯХ}

Напомним, что для систем натурального вида гамильтониан $H$ является суммой положительно-определенной кинетической энергии $T$ и потенциальной энергии $V$. Например, на симплектическом многообразии $M \simeq \mathbb{R}^{2 n}$, т.е. в евклидовом пространстве, функция Гамильтона имеет вид

$$
H=T+V=\sum_{i=1}^{n} p_{i}^{2}+V(q) .
$$

Здесь $p$ и $q$ - канонические координаты, так что скобки Пуассона для произвольных функций $f, g \in C^{\infty}(M)$ определяются соотношениями

$$
\{f, g\}=\sum_{i=1}^{n}\left(\frac{\partial f}{\partial p_{i}} \frac{\partial g}{\partial q_{i}}-\frac{\partial f}{\partial q_{i}} \frac{\partial g}{\partial p_{i}}\right) .
$$

Соответствующий тензор Пуассона

$$
P=\left(\begin{array}{cc}
0 & I \\
-I & 0
\end{array}\right), \quad I=\operatorname{diag}(1, \ldots, 1),
$$

называется каноническим тензором.

В данной работе рассматривается вопрос о построении интегрируемых систем натурального вида на $\omega N$-многообразиях, которые являются частным случаем бигамильтоновых многообразий общего положения [1], [3]-[5].

2.1. $\omega N$-многообразия. Введем некоторые определения.

ОПРеДЕлЕНиЕ 1. Тензоры Пуассона $P$ и $P^{\prime}$ называются совместными, если любая их линейная комбинация $\widetilde{P}=P+\lambda P^{\prime}$ также является тензором Пуассона.

Здесь и всюду далее подразумевается, что тензоры $P$ и $P^{\prime}$ независимы.

ОПРЕДЕЛЕНиЕ 2. Если один из тензоров Пуассона невырожден, то бигамильтоново многообразие $M$ называется $\omega N$-многообразием.

Без ограничения общности можно предположить, что невырожден первый тензор $P$. В этом случае на $\omega N$-многообразии $M$ определена и симплектическая форма $\omega=P^{-1}$, и оператор

$$
N=P^{\prime} P^{-1},
$$

который называют оператором рекурсии (формально) [3], [4], а также тензором Нийенхейса [5] или наследующим оператором [6]. Бигамильтоново многообразие $M$, снабженное оператором рекурсии $N$, принято называть многообразием ПуассонаНийенхейса. 
В рассматриваемом нами случае для построения оператора $N(2.4)$ необходимо найти тензор Пуассона, совместный с каноническим тензором (2.3). Если $z=$ $(q, p)=\left(q_{1}, \ldots, q_{n}, p_{1}, \ldots, p_{n}\right)$, то скобки Пуассона, отвечающие тензору Пуассона $P^{\prime}$, определяются следующим образом:

$$
\{f(z), g(z)\}^{\prime}=\sum_{i, k=1}^{2 n} P_{i k}^{\prime}(z) \frac{\partial f(z)}{\partial z_{i}} \frac{\partial g(z)}{\partial z_{k}} .
$$

В общем случае элементы $P_{i k}^{\prime}(z)$ тензора $P^{\prime}$ являются функциями координат $q$ и импульсов $p$, которые удовлетворяют системе алгебраических уравнений (кососимметричность)

$$
P_{i j}^{\prime}=-P_{j i}^{\prime}, \quad i, j=1,2, \ldots, 2 n,
$$

и системе дифференциальных уравнений

$$
\sum_{m=1}^{2 n}\left(P_{i m}^{\prime} \frac{\partial P_{j k}^{\prime}}{\partial z_{m}}+P_{j m}^{\prime} \frac{\partial P_{k i}^{\prime}}{\partial z_{m}}+P_{k m}^{\prime} \frac{\partial P_{i j}^{\prime}}{\partial z_{m}}\right)=0, \quad i, j, k=1,2, \ldots, 2 n,
$$

которые следуют из тождества Якоби

$$
\left\{f,\{g, h\}^{\prime}\right\}^{\prime}+\left\{g,\{h, f\}^{\prime}\right\}^{\prime}+\left\{h,\{f, g\}^{\prime}\right\}^{\prime}=0
$$

для произвольных функций $f, g$ и $h \in C^{\infty}(M)$.

Так как искомый тензор $P^{\prime}$ должен быть совместен с тензором $P(2.3)$, то функции $\widetilde{P}_{i j}(z)=P_{i j}(z)+\lambda P_{i j}^{\prime}(z)$ должны удовлетворять дифференциальным уравнениям

$$
\sum_{m=1}^{n}\left(\widetilde{P}_{i m} \frac{\partial \widetilde{P}_{j k}}{\partial z_{m}}+\widetilde{P}_{j m} \frac{\partial \widetilde{P}_{k i}}{\partial z_{m}}+\widetilde{P}_{k m} \frac{\partial \widetilde{P}_{i j}}{\partial z_{m}}\right)=0, \quad i, j, k=1,2, \ldots, 2 n,
$$

которые следуют из тождества Якоби для скобок Пуассона $\{\cdot, \cdot\}_{\lambda}=\{\cdot, \cdot\}+\lambda\{\cdot, \cdot\}^{\prime}$, где $\lambda$ - произвольный числовой параметр.

Любое решение $P^{\prime}(z)$ уравнений $(2.5),(2.7)$ определяет тензор $N(2.4)$, с помощью которого систему уравнений $(2.5),(2.7)$ можно записать в инвариантном виде.

Теорема 1 [7], [1]. Для пуассонова пучка $\widetilde{P}=P+\lambda P^{\prime}$ кручение Нийенхейса оператора рекурсии $N$ равно нулю,

$$
T_{N}(X, Y)=[N X, N Y]-N([N X, Y]+[X, N Y]-N[X, Y])=0,
$$

для любых векторных полей $X, Y$ на $\omega N$-многообразии $M$.

Всюду далее мы будем использовать частные решения системы уравнений (2.5), (2.7) или эквивалентной ей системы (2.8).

ОПРЕДЕЛЕНИЕ 3 . Пусть $M$ является $\omega N$-многообразием размерности $2 n$. Если в любой точке $z \in M$ оператор рекурсии $N$ имеет $n$ различных собственных значений $\lambda_{1}, \ldots, \lambda_{n}$, то такое многообразие $M$ называется полупростым. Если собственные значения $\lambda_{i}$ оператора рекурсии функционально независимы, то многообразие $M$ называется регулярным. 
Уравнения $(2.5),(2.7)$ имеют бесконечно много решений. Поэтому далее мы будем решать эти уравнения в классе функций $P_{i j}^{\prime}(q, p)$, полиномиальных по импульсам $p$, что позволит нам найти достаточно большое, но все же конечное число решений.

ПримеР 1. Два кососимметричных тензора

$$
P^{\prime}=\left(\begin{array}{cccc}
0 & 0 & 0 & q_{1} \\
* & 0 & q_{1} & 2 q_{2} \\
* & * & 0 & -p_{1} \\
* & * & * & 0
\end{array}\right), \quad P^{\prime}=\left(\begin{array}{cccc}
0 & 0 & a_{1}-q_{1}^{2} & -q_{1} q_{2} \\
* & 0 & -q_{1} q_{2} & a_{2}-q_{2}^{2} \\
* & * & 0 & -q_{1} p_{2}+q_{2} p_{1} \\
* & * & * & 0
\end{array}\right)
$$

являются тензорами Пуассона, совместными с каноническим тензором $P(2.3)$. Соответствующие операторы $N(2.4)$ обладают нулевым кручением Нийенхейса и их собственные значения функционально независимы. Таким образом, тензоры (2.9) задают структуру регулярного полупростого $\omega N$-многообразия в $M=\mathbb{R}^{4}$.

2.2. Координаты Дарбу-Нийенхейса. Оператор рекурсии существует практически для всех интегрируемых по Лиувиллю динамических систем [8]. В силу этого классификация пуассоновых пучков $P+\lambda P^{\prime}$ должна практически повторять классификацию интегрируемых систем, которая имеет значительно более длинную историю (см. [1], [2], [9]). В основе этой взаимосвязи лежит тот факт, что оператор $N$ порождает переменные разделения для соответствующих бигамильтоновых интегрируемых систем.

ОПРЕДЕЛЕНИЕ 4 . Локальные координаты $\left(x_{i}, y_{i}\right)$ на $\omega N$-многообразии называются координатами Дарбу-Нийенхейса, если они являются координатами Дарбу относительно симплектической формы

$$
\omega=P^{-1}=\sum_{i=1}^{n} d y_{i} \wedge d x_{i}
$$

и диагонализуют оператор рекурсии

$$
N=\sum_{i=1}^{n} \lambda_{i}\left(\frac{\partial}{\partial x_{i}} \otimes d x_{i}+\frac{\partial}{\partial y_{i}} \otimes d y_{i}\right),
$$

что эквивалентно соотношениям $\left\{x_{i}, y_{j}\right\}=\delta_{i j},\left\{x_{i}, y_{j}\right\}^{\prime}=\lambda_{i} \delta_{i j}$.

Из определения (2.10) следует, что

$$
N^{*} d x_{i}=\lambda_{i} d x_{i}, \quad N^{*} d y_{i}=\lambda_{i} d y_{i},
$$

где $N^{*}$ - оператор, сопряженный оператору $N$. Так как для оператора $N$ кручение Нийенхейса $T_{N}$ равно нулю, то

$$
N^{*} d \lambda_{i}=\lambda_{i} d \lambda_{i}
$$

В силу этого собственные значения $\lambda_{i}$ оператора рекурсии можно рассматривать как координаты Дарбу-Нийенхейса специального вида, которые всегда существуют, если многообразие $\mathcal{M}$ регулярно. 
Теорема 2 [3], [4]. Если в окрестности точки $z \in M$ регулярного $\omega N$-многообразия собственные значения $\lambda_{i}$ оператора рекурсии $N$ различны, то в этой окрестности можно с помощью квадратур определить $n$ функиий $\mu_{1}, \ldots, \mu_{n}$, которые вместе с собственными значениями $\lambda_{1}, \ldots, \lambda_{n}$ являются координатами ДарбуНийенхейса.

Так как $\lambda_{i}$ являются собственными значениями оператора рекурсии (как минимум кратности два), то их легко найти в виде корней минимального характеристического полинома оператора $N$

$$
\Delta(\lambda)=(\operatorname{det}(N-\lambda I))^{1 / 2}=\prod_{j=1}^{n}\left(\lambda-\lambda_{j}\right) .
$$

Сопряженные координаты $\mu_{j}$ находятся с помощью интегрирования соотношений (2.11), в которых необходимо положить $x_{i}=\lambda_{i}$ и $y_{i}=\mu_{i}$.

ПримеР 2. Для первого тензора $P^{\prime}$ в $(2.9)$ минимальный характеристический полином оператора рекурсии $N$ имеет вид

$$
\Delta(\lambda)=\lambda^{2}-q_{2} \lambda-\frac{q_{1}}{4}=\left(\lambda-\lambda_{1}\right)\left(\lambda-\lambda_{2}\right) .
$$

Соответствующие координаты Дарбу-Нийенхейса специального вида

$$
\lambda_{1,2}=\frac{1}{2}\left(q_{2} \pm \sqrt{q_{1}^{2}+q_{2}^{2}}\right), \quad \mu_{1,2}=p_{2}-\frac{p_{1}}{q_{1}}\left(q_{2} \pm \sqrt{q_{1}^{2}+q_{2}^{2}}\right)
$$

являются параболическими координатами на плоскости и сопряженными им импульсами. Для второго тензора $P^{\prime}$ в $(2.9)$ соответствующий минимальный полином

$$
\Delta(\lambda)=\lambda^{2}+\left(q_{1}^{2}+q_{2}^{2}-a_{1}-a_{2}\right) \lambda+a_{1} a_{2}-a_{2} q_{1}^{2}-a_{1} q_{2}^{2}=\left(\lambda-\lambda_{1}\right)\left(\lambda-\lambda_{2}\right)
$$

является производящей функцией эллиптических координат $\lambda_{1,2}$ на плоскости.

2.3. Интегрируемые системы в методе Якоби. Согласно методу Якоби переменные разделения, совпадающие в нашем случае с координатами Дарбу-Нийенхейса, можно использовать для построения сколь угодно большого семейства интегрируемых систем.

Теорема 3. Произведение $n$ одномерных лагранжевых подмногообразий

$$
\mathcal{C}_{i}: \quad \Phi_{i}\left(\mu_{i}, \lambda_{i} ; \alpha_{1}, \ldots, \alpha_{n}\right)=0 \quad \operatorname{ma\kappa ux,~umo~} \quad \operatorname{det}\left\|\frac{\partial \Phi_{i}\left(\mu_{i}, \lambda_{i}\right)}{\alpha_{j}}\right\| \neq 0,
$$

является $n$-мерным лагранжевым подмногообразием в $M$. Решения разделенных уравнениц $\Phi_{i}=0$ (2.12) относительно параметров $\alpha_{k}$ являются функиионально независимыми функциями $H_{k}=\alpha_{k}(\mu, \lambda)$ в инволюции.

Основным недостатком метода Якоби является тот факт, что мы априори не знаем, какие разделенные уравнения $\Phi_{i}\left(\lambda_{i}, \mu_{i}, \alpha\right)=0$ необходимо использовать для получения интегрируемых систем натурального вида или других физически интересных динамических систем.

Для построения бигамильтоновых интегрируемых систем необходимого нам типа можно использовать следующие известные факты. 
ТЕОРема 4 [3], [4]. Если $M$ является регулярным $\omega N$-многообразием размерности $2 n$ и $\left\{H_{1}, \ldots, H_{n}\right\}$ - семейство независимых функиий на $M$, то следующие утверждения эквивалентнь:

1) функиии $\left\{H_{1}, \ldots, H_{n}\right\}$ находятся в биинволючии (1.1);

2) лагранжсево расслоение $\mathcal{F}$, отвечающее функииям $\left\{H_{1}, \ldots, H_{n}\right\}$, допускает разделение переменных в координатах Дарбу-Нийенхейса;

3) распределение $\mathcal{D}$, образованное векторными полями $X_{H_{k}}$, лагранжево относительно $\omega=P^{-1}$ и инвариантно относительно действия оператора $N$.

Инвариантность распределения $\mathcal{D}$ относительно действия оператора $N$ означает, что существует контрольная матрица $F$, собственные значения которой равны собственным значениям оператора рекурсии $\lambda_{i}$, такая, что

$$
N^{*} d H_{j}=\sum_{k=1}^{n} F_{j k} d H_{k}, \quad i=1,2, \ldots, n .
$$

В переменных Дарбу-Нийенхейса уравнения (2.13) совпадают с известным критерием Леви-Чивита [9], в котором описываются гамильтоновы системы, допускающие разделение переменных в данной системе координат.

Обычно критерий Леви-Чивита используют для нахождения переменных разделения, т.е. подставляют в уравнения (2.13) конкретные интегралы движения $H_{i}$ и затем решают полученные уравнения относительно $N$ [3], [4], [10], [11]. В данной работе мы будем решать обратную задачу. Подставляя неизвестный оператор $N$ и неизвестный гамильтониан натурального вида $H=H_{1}(2.1)$ в соотношения (2.8) и (2.13), мы получим систему уравнений

$$
T_{N}=0, \quad N^{*} d H=F d H .
$$

Эту систему уравнений мы и предлагаем решать относительно потенциала $V(q)$, интегралов движения $H_{2}(p, q), \ldots, H_{n}(p, q)$ и функций $N_{i j}(p, q)$.

Предложенный метод наследует основной недостаток метода Якоби, так как контрольная матрица $F$ в уравнениях (2.14) остается неопределенной, так же как и разделенные уравнения $\Phi_{i}=0$ в методе Якоби. Одной из возможностей решения этой проблемы является использование контрольных матриц $F$ специального вида.

ПримеР 3. Если $H_{k}=\operatorname{tr} N^{k} /(2 k)$, то контрольная матрица в уравнении $(2.13)$ имеет вид

$$
F=\left(\begin{array}{ccccc}
0 & 1 & 0 & \ldots & 0 \\
0 & 0 & 1 & \ldots & 0 \\
\ldots \ldots \ldots \ldots \ldots \ldots \ldots \ldots \ldots \\
0 & 0 & 0 & \ldots & 1 \\
\sigma_{n} & \sigma_{n-1} & \sigma_{n-2} & \ldots & \sigma_{1}
\end{array}\right)
$$

где $\sigma_{k}$ - элементарные симметрические полиномы степени $k$ от собственных значений $\lambda_{i}$ оператора $N$. В этом случае уравнения (2.13) становятся стандартными рекуррентными соотношениями

$$
N^{*} d H_{i}=d H_{i+1} \quad \text { или } \quad P^{\prime} d H_{i}=P d H_{i+1} .
$$


Интегралы движения $H_{i}$ образуют цепочку Ленарда-Магри, а соответствующие векторные поля $X_{H_{i}}$ являются бигамильтоновыми [1], [4], [12].

2.4. Однородные системы Штеккеля. Рассмотрим специальный класс разделенных уравнений вида

$$
\Phi_{i}\left(\mu_{i}, \lambda_{i}, \alpha_{1}, \ldots, \alpha_{n}\right)=\sum_{j=1}^{n} S_{i j}\left(\lambda_{i}\right) \alpha_{j}-s_{i}\left(\mu_{i}, \lambda_{i}\right)=0, \quad H_{i}=\alpha_{i} .
$$

Здесь $S$ и $s$ называются матрицей и вектором Штеккеля, для которых $\operatorname{det} S \neq 0$ и элементы $S_{i j}, s_{i}$ зависят только от пары сопряженных координат $\lambda_{i}$ и $\mu_{i}$.

Теорема 5 [3], [4]. Предположим, что семейство $\left\{H_{1}, \ldots, H_{n}\right\}$ независимых интегралов движения в биинволючии определяет билагранжево расслоение регулярного полупростого $\omega N$-многообразия. Если матрица $F$ в (2.13) удовлетворяет соотношениям

$$
N^{*} d F=F d F, \quad \text { m.e. } \quad N^{*} d F_{i j}=\sum_{k=1}^{n} F_{i k} d F_{k j}, \quad i, j=1,2, \ldots, n,
$$

то интегралы движения $\left\{H_{1}, \ldots, H_{n}\right\}$ допускают штеккелевское разделение переменных (2.16) относительно координат Дарбу-Нийенхейса, а матрица Штеккеля $S$ находится из уравнения

$$
F=S^{-1} \Lambda S, \quad \Lambda=\operatorname{diag}\left(\lambda_{1}, \ldots, \lambda_{n}\right) .
$$

Подставляя оператор $N$ и гамильтониан натурального вида $H=H_{1}(2.1)$ в соотношения $(2.8),(2.13)$ и $(2.17)$, мы получим расширенную систему дифференциальных уравнений

$$
T_{N}=0, \quad N^{*} d H=F d H, \quad N^{*} d F=F d F
$$

для неизвестных функций $V(q), H_{2}(p, q), \ldots, H_{n}(p, q), N_{i j}(p, q)$ и $F_{i j}(p, q)$.

Систему уравнений (2.18) также пока невозможно исследовать в общем виде. Дело в том, что хотя мы и зафиксировали необходимый нам вид функции Гамильтона (2.1) и класс разделенных уравнений (2.16), но входящий в них вектор Штеккеля $s$ остается по-прежнему неопределенным.

Поэтому далее мы будем рассматривать однородные системы Штеккеля, для которых матрица Штеккеля и разделенные уравнения имеют вид

$$
S=\left(\begin{array}{lllr}
\lambda_{1}^{n-1} & \ldots & \lambda_{1} & 1 \\
\ldots \ldots & \ldots & \ldots & \ldots \\
\lambda_{n}^{n-1} & \ldots & \lambda_{n} & 1
\end{array}\right), \quad \Phi_{i}=\sum_{k=0}^{n-1} \alpha_{k} \lambda_{i}^{k}=s_{i}\left(\mu_{i}, \lambda_{i}\right)
$$

В этом случае контрольная матрица равна

$$
F=S^{-1} \Lambda S=\left(\begin{array}{ccccc}
\sigma_{1} & 1 & 0 & \ldots & 0 \\
\sigma_{2} & 0 & 1 & \ldots & 0 \\
\ldots \ldots & \ldots & \ldots & \ldots \\
\sigma_{n-1} & 0 & 0 & \ldots & 1 \\
\sigma_{n} & 0 & 0 & \ldots & 0
\end{array}\right)
$$


где $\sigma_{j}$ - симметрические полиномы от собственных значений $\lambda_{j}$ оператора $N$

$$
\Delta(\lambda)=\lambda^{n}-\sigma_{1} \lambda^{n-1}-\sigma_{2} \lambda^{n-2}-\cdots-\sigma_{n} .
$$

Решая разделенные уравнения (2.16) относительно постоянных $\alpha_{1}, \ldots, \alpha_{n}$, можно построить соответствующие им интегралы движения $H_{i}$ :

$$
H_{i}=\sum_{j=1}^{n} \frac{\partial \sigma_{j}}{\partial \lambda_{j}} \frac{s_{i}\left(\mu_{i}, \lambda_{i}\right)}{\prod_{i \neq k}\left(\lambda_{i}-\lambda_{k}\right)} .
$$

Соответствующие векторные поля $X_{H_{i}}$ называются квазибигамильтоновыми полями пфаффовского типа [3], [4].

Напомним, что в общем случае матрица Штеккеля $S$ совпадает с транспонированной матрицей Бриль-Нётер, которая является производной отображения Абеля, т.е. является линейным отображением из касательного пространства к лагранжеву подмногообразию данной интегрируемой системы в касательное пространство якобиана некоторой алгебраической кривой [13].

В нашем случае матрица $S$ вида (2.19) определена с помощью канонического базиса (базиса Веронезе) в пространстве голоморфных дифференциалов на гиперэллиптической кривой $\mathcal{C}[13]$.

ГипотезА. Для интегрируемых систем с контролъной матрицей $F$ вида (2.20) билагранжево расслоение, отвечающее интегралам движения $H_{i}(2.22)$, представимо в виде произведения гиперэллиптических кривых $\mathcal{C}_{i}$, задаваемъх разделеннъми уравнениями (2.19).

Согласно этой гипотезе, выбирая контрольную матрицу $F$ вида $(2.20)$, мы тем самым де-факто фиксируем и вектор Штеккеля $s$, так как алгебраическая кривая, отвечающая разделенным уравнениям, должна быть гиперэллиптической кривой.

Эта гипотеза верна для всех известных на данный момент интегрируемых систем, обладающих контрольной матрицей вида (2.20). Кроме того, для всех известных примеров интегрируемых систем на более общих не гиперэллиптических кривых $\mathcal{C}_{i}$ соответствующая матрица $F$ имеет другой вид [3], [4].

ПримеР 4. Рассмотрим интегрируемую систему на плоскости, допускающую разделение переменных в полярных координатах $\left(r, \phi, p_{r}, p_{\phi}\right)$. Соответствующий тензор Пуассона

$$
P^{\prime}=\left(\begin{array}{cccc}
0 & 0 & r^{2} & 0 \\
* & 0 & 0 & 0 \\
* & * & 0 & 0 \\
* & * & * & 0
\end{array}\right)
$$

задает структуру регулярного, но не полупростого $\omega N$-многообразия в $M=\mathbb{R}^{4}$. Для интегралов движения

$$
H_{1}=p_{r}^{2}+\frac{p_{\phi}^{2}}{r^{2}}+V(r), \quad H_{2}=p_{\phi}
$$


контрольная матрица и разделенные уравнения имеют вид

$$
\begin{gathered}
F=\left(\begin{array}{cc}
r^{2} & 0 \\
0 & 0
\end{array}\right), \\
\Phi_{1}=p_{r}^{2}+\frac{\alpha_{2}^{2}}{r^{2}}+V(r)-\alpha_{1}=0, \quad \Phi_{2}=p_{\phi}-\alpha_{2}=0 .
\end{gathered}
$$

Если $V(r)$ - полином, то первое из разделенных уравнений $\Phi_{1}$ задает гиперэллиптическую кривую на плоскости, а второе уравнение $\Phi_{2}-$ прямую.

\section{3. БИГАМИЛЬТОНОВЫ СИСТЕМЫ $\mathbf{B} \mathbb{R}^{2 n}$}

В настоящее время известны два больших класса бигамильтоновых систем натурального вида в евклидовом пространстве $\mathbb{R}^{2 n}$. Изучению этих систем посвящено достаточно большое количество работ (см. [3], [4], [12], [14]-[16] и приведенную там литературу). В этом разделе мы лишь кратко опишем эти два класса интегрируемых систем.

3.1. Системы Штеккеля: квадратичные интегралы. Предположим, что $M=T^{*} Q$ и на конфигурационном пространстве $Q \simeq \mathbb{R}^{n}$ определен конформный тензор Киллинга $L$ градиентного типа, обладающий нулевым кручением Нийенхейса и различными собственными значениями $\lambda_{1}, \ldots, \lambda_{n}[12]$, [14].

В этом случае оператор $N$ является полным поднятием тензора $L$ на фазовое пространство $M=T^{*} Q$ :

$$
N \frac{\partial}{\partial q^{k}}=\sum_{i=1}^{n} L_{k}^{i} \frac{\partial}{\partial q^{i}}+\sum_{i j} p_{j}\left(\frac{\partial L_{i}^{j}}{\partial q^{k}}-\frac{\partial L_{k}^{j}}{\partial q^{i}}\right) \frac{\partial}{\partial p_{i}}, \quad N \frac{\partial}{\partial p_{k}}=\sum_{i=1}^{n} L_{i}^{k} \frac{\partial}{\partial p_{i}} .
$$

Соответствующий второй тензор Пуассона

$$
P^{\prime}=N P=\left(\begin{array}{cc}
0 & -L_{i}^{j} \\
L_{i}^{j} & \left(\frac{\partial L_{j}^{k}}{\partial q_{i}}-\frac{\partial L_{i}^{k}}{\partial q_{j}}\right) p_{k}
\end{array}\right)
$$

является линейным полиномом по импульсам $p$.

ПримеР 5. В пространстве $M=\mathbb{R}^{4}$ существует всего четыре подобных линейных тензора $P^{\prime}$ : тензоры $(2.9)$, отвечающие эллиптическим и параболическим координатам, тензор (2.23), отвечающий полярным координатам, и числовой кососимметричный тензор, отвечающий декартовым координатам [14].

Для тензора Пуассона $P^{\prime}$ вида (3.1) и натурального гамильтониана $H_{1}=T+V$ (2.1) система уравнений (2.14) распадается на два уравнения

$$
d\left(\mathcal{L}_{X_{T}} \theta^{\prime}-T d \sigma_{1}\right)=0, \quad d\left(\mathcal{L}_{X_{V}} \theta^{\prime}-V d \sigma_{1}\right)=0,
$$

определяющих тензор $L$ и потенциал $V$, а также систему рекуррентных уравнений для определения интегралов движения

$$
N^{*} d H_{i}=d H_{i+1}+\sigma_{i} d H_{1}, \quad i=2,3, \ldots, n,
$$


которые необходимо решать, начиная с $i=n$ при условии $H_{n+1}=0$. В уравнения (3.2) входит производная Ли $\mathcal{L}$ вдоль векторного поля $X$ и деформация стандартной формы Лиувилля $\theta=\sum_{j} p_{j} d q^{j}$ вида $\theta^{\prime}=\sum_{i, j=1}^{n} L_{j}^{i} p_{i} d q^{j}$.

В работах [11] предложено программное обеспечение, которое позволяет эффективно решать уравнения вида (3.2) и (3.3) на различных римановых многообразиях $Q$ положительной кривизны, в том числе и на евклидовых пространствах.

ЗАмечание 1. Поскольку тензор Пуассона $P^{\prime}$ является линейной функцией от импульсов $p_{j}$ специального вида, а функции $\sigma_{i}$ зависят только от координат $q$, то согласно уравнениям (3.3) для интегрируемых систем натурального вида все интегралы движения $H_{2}, \ldots, H_{n}$ будут не более чем квадратичными полиномами по импульсам.

ЗАмечаниЕ 2. Поскольку собственные значения оператора $N$ совпадают с собственными значениями $\lambda_{i}\left(q_{1}, \ldots, q_{n}\right)$ тензора $L$, которые являются функциями на конфигурационном пространстве $Q$, то соответствующие интегрируемые системы допускают разделение переменных в ортогональных криволинейных системах координат.

3.2. Цепочки Тоды. Бигамильтонова структура открытых цепочек Тоды $\mathcal{A}_{n^{-}}$ типа с гамильтонианом

$$
H=\frac{1}{2} \sum_{i=1}^{n} p_{i}^{2}+\sum_{i=1}^{n-1} e^{2\left(q_{i}-q_{i+1}\right)}
$$

активно исследовалась в ряде работ начиная с 1979 года (см. [16] и приведенную там литературу). Второй оператор Пуассона

$$
P^{\prime}=\sum_{i=1}^{n-1} 2 e^{2\left(q_{i}-q_{i+1}\right)} \frac{\partial}{\partial p_{i+1}} \wedge \frac{\partial}{\partial p_{i}}+\sum_{i=1}^{n} p_{i} \frac{\partial}{\partial q_{i}} \wedge \frac{\partial}{\partial p_{i}}+\frac{1}{2} \sum_{i<j}^{n} \frac{\partial}{\partial q_{j}} \wedge \frac{\partial}{\partial q_{i}}
$$

является линейной функцией от импульсов [16].

Для открытых цепочек Тоды, отвечающих классическим корневым системам $\mathcal{B C}_{n}$ и $\mathcal{D}_{n}$-типа, второй тензор Пуассона $\widetilde{P}^{\prime}=P^{\prime} P^{-1} P^{\prime}+K(p, q)$ является квадратичным полиномом по импульсам [15]. Здесь $P^{\prime}-$ линейный по импульсам тензор для цепочки Тоды $\mathcal{A}_{n}$-типа (3.4), а дополнительное слагаемое $K(p, q)$ является линейной функцией от импульсов $p_{1}$ и $p_{n}$, которая зависит от типа корневой системы.

В следующем разделе мы предъявим обобщение известных квадратичных тензоров Пуассона [15], которое связано с деформацией цепочек Тоды, предложенной Иноземцевым [17].

\section{4. БИГАМИЛЬТОНОВЫ СИСТЕМЫ НА ПЛОСКОСТИ}

В данном разделе мы используем функцию Гамильтона натурального вида $H(2.1)$ и полиномиальную по импульсам подстановку для тензора Пуассона $P^{\prime}$ в уравнения (2.14) и опишем все нетривиальные физические решения полученных уравнений при $n=2$. 
ОПРеДЕЛЕниЕ 5. Решение уравнений (2.14) называется тривиалъным, если интегралы движения функционально зависимы или постоянны, если $V=$ const и если $V(q)$ является комплексной функцией.

Мы ограничимся рассмотрением только полупростых и регулярных $\omega N$-многообразий, так как в противном случае нам не известны ни переменные разделения, ни форма разделенных уравнений и, следовательно, класс соответствующих интегрируемых систем слишком широк.

ПримеР 6. Подставляя тензор Пуассона $P^{\prime}$, не зависящий от импульсов, и гамильтониан $H_{1}=p_{1} p_{2}+V\left(q_{1}, q_{2}\right)$ в $(2.14)$, можно получить следующее решение:

$$
\begin{gathered}
P^{\prime}=\left(\begin{array}{cccc}
0 & 0 & 0 & 0 \\
* & 0 & f\left(q_{1}\right) & 0 \\
* & * & 0 & 0 \\
* & * & * & 0
\end{array}\right), \\
H_{1}=p_{1} p_{2}+\frac{q_{2}}{4 C_{1} q_{1}}+f\left(q_{1}\right), \quad H_{2}=\sqrt{q_{1}} e^{C_{1} p_{2}^{2}} .
\end{gathered}
$$

Такие решения уравнений (2.14) далее не рассматриваются.

Интегрируемые системы натурального вида на плоскости являются одними из наиболее исследованных семейств интегрируемых систем [2], [9]. Поэтому, решая уравнения (2.14) при $n=2$, мы можем только построить неизвестные ранее операторы рекурсии для уже известных интегрируемых систем. Тем не менее нам удалось найти две новые интегрируемые системы с псевдоевклидовой метрикой.

4.1. Тензор Пуассона, линейный по импульсам. Рассмотрим следующий анзац для тензора Пуассона:

$$
P^{\prime}=\left(\begin{array}{cccc}
0 & h(q) & c_{1} p_{1}+c_{2} p_{2} & c_{3} p_{3}+c_{4} p_{4} \\
* & 0 & c_{5} p_{1}+c_{6} p_{2} & c_{7} p_{1}+c_{8} p_{2} \\
* & * & 0 & f(q) \\
* & * & * & 0
\end{array}\right),
$$

где $f$ и $h$ - функции от координат $q$, а $c_{k}$ - числовые параметры.

ПреДЛОЖЕНИЕ 1. Решения уравнений (2.5), (2.7) вида (4.1) существуют тогда и только тогда, когда функции $h(q)$ и $f(q)$ имеют вид

$$
\begin{aligned}
& \text { 1) } h\left(q_{1}, q_{2}\right)=\text { const, } \quad f\left(q_{1}, q_{2}\right)=e^{q_{1}} \text {, } \\
& \text { 2) } h\left(q_{1}, q_{2}\right)=q_{1}, \quad f\left(q_{1}, q_{2}\right)=q_{1}^{\alpha} \text {, }
\end{aligned}
$$

c точностъю до преобразований Галилея $q \rightarrow q^{\prime}=A q+b$. 
В первом случае тензор Пуассона, совместный с каноническим, имеет вид

$$
P_{1}^{\prime}=\left(\begin{array}{cccc}
0 & -\frac{2\left(c_{1}^{2}+c_{2} c_{3}\right)}{c_{3}} & c_{1} p_{1}+c_{2} p_{2} & c_{3} p_{1}-c_{1} p_{2} \\
* & 0 & c_{2} p_{1}-\frac{c_{1} c_{2}}{c_{3}} p_{2} & -c_{1} p_{1}+\frac{c_{2} c_{3}+2 c_{1}^{2}}{c_{3}} p_{2} \\
* & * & 0 & b e^{q_{1}} \\
* & * & * & 0
\end{array}\right)
$$

Во втором случае существуют два тензора Пуассона с произвольным показателем $\alpha$

$$
P_{2}^{\prime}=\left(\begin{array}{cccc}
0 & q_{1} & -\frac{(\alpha+1)}{2} p_{2} & 0 \\
* & 0 & \frac{(1-\alpha)}{2} p_{1}+a p_{2} & -\frac{(\alpha+1)}{2} p_{2} \\
* & * & 0 & b q_{1}^{\alpha} \\
* & * & * & 0
\end{array}\right), \quad P_{3}^{\prime}=\left(\begin{array}{cccc}
0 & q_{1} & 0 & 0 \\
* & 0 & p_{1}+a p_{2} & -(\alpha+1) p_{2} \\
* & * & 0 & b q_{1}^{\alpha} \\
* & * & * & 0
\end{array}\right)
$$

и один тензор Пуассона с $\alpha=-3$

$$
P_{4}^{\prime}=\left(\begin{array}{cccc}
0 & q_{1} & c_{1} p_{1}+c_{2} p_{2} & -\frac{c_{1}^{2}}{c_{2}} p_{1}-c_{1} p_{2} \\
* & 0 & \left(c_{2}+1\right) p_{1}+\frac{c_{2}\left(c_{2}-1\right)}{c_{1}} p_{2} & -c_{1} p_{1}+\left(2-c_{2}\right) p_{2} \\
* & * & 0 & b q_{1}^{-3} \\
* & * & * & 0
\end{array}\right)
$$

Решения расширенной системы уравнений (2.14) описываются следующими двумя предложениями.

ПреДЛОЖЕНИЕ 2. Если тензор Пуассона $P^{\prime}$ имеет вид (4.1), то система уравнений (2.14) с матрицей $F$ (2.15) имеет единственное нетривиальное решение

$$
P^{\prime}=\left(\begin{array}{cccc}
0 & a & p_{1} & 0 \\
* & 0 & 0 & p_{2} \\
* & 0 & 0 & b e^{\left(q_{2}-q_{1}\right) / a} \\
* & * & * & 0
\end{array}\right), \quad H_{1}=p_{1}^{2}+p_{2}^{2}+2 a b e^{\left(q_{2}-q_{1}\right) / a}
$$

Второй интеграл движения $H_{2}=\left(p_{1}+p_{2}\right) H_{1}-\left(p_{1}+p_{2}\right)^{3} / 3$ формально является полиномом третьей степени по импульсам.

Это решение системы уравнений (2.14) совпадает с открытой цепочкой Тоды $\mathcal{A}_{2}$-типа.

ПРЕДЛОЖЕНИЕ 3. Нетривиальнъе решения уравнений (2.5), (2.7) вида (4.1) и уравнений (2.13) с контрольной матрицей $F$ (2.20) при

$$
H_{1}=p_{1}^{2}+p_{2}^{2}+V\left(q_{1}, q_{2}\right), \quad H_{2}=H_{2}\left(p_{1}, p_{2}, q_{1}, q_{2}\right)
$$


имеют вид

$$
\begin{aligned}
P_{1}^{\prime} & =\left(\begin{array}{cccc}
0 & -2 & p_{2} & 3 p_{1} \\
* & 0 & p_{1} & p_{2} \\
* & * & 0 & b e^{q_{1}} \\
* & * & -b e^{q_{1}} & 0
\end{array}\right), \quad V_{1}=-\frac{2 b}{3} e^{q_{1}}+C_{1} e^{-q_{1} / 2+\sqrt{3} q_{2} / 2}+C_{2} e^{-q_{1} / 2-\sqrt{3} q_{2} / 2}, \\
P_{2}^{\prime} & =\left(\begin{array}{cccc}
0 & q_{1} & -2 p_{2} / 3 & 0 \\
* & 0 & p_{1} / 3 & -2 p_{2} / 3 \\
* & * & 0 & b q_{1}^{1 / 3} \\
* & * & * & 0
\end{array}\right), \quad V_{2}=\frac{b\left(3 q_{1}^{2}+4 q_{2}^{2}\right)+C_{1} q_{2}+C_{2}}{4 q_{1}^{2 / 3}}, \\
P_{3}^{\prime} & =\left(\begin{array}{cccc}
0 & q_{1} & 0 & 0 \\
* & 0 & p_{1} & -2 p_{2} \\
* & * & 0 & b q_{1} \\
* & * & * & 0
\end{array}\right), \quad V_{3}=b\left(\frac{q_{1}^{2}}{4}+q_{2}^{2}\right)+C_{1} q_{2}+\frac{C_{2}}{q_{1}^{2}}, \\
P_{4}^{\prime} & =\left(\begin{array}{cccc}
0 & q_{1} & 0 & 4 p_{1} \\
* & 0 & p_{1} & 2 p_{2} \\
* & * & 0 & b / q_{1}^{3} \\
* & * & * & 0
\end{array}\right), \quad V_{4}=\frac{b}{4 q_{1}^{2}}+C_{1} q_{2}^{2}+C_{2} q_{2}-C_{3} q_{1}^{2},
\end{aligned}
$$

где $C_{j}$ - произвольные постоянные, возникающие при интегрировании дифференциалъных уравнений.

Соответствующие вторые интегралы движения $H_{2}$ являются полиномами третьей степени по импульсам, которые мы не будем выписывать явно, так как первое решение отвечает трехчастичной периодической цепочке Тоды в системе центра масс [2], второе решение известно как система Холта [2], [18], третье и четвертое решения можно рассматривать как системы типа Калоджеро [2], [9]. Это означает, что полученные нами интегралы движения совпадают с известными интегралами движения, полученными, например, с помощью представлений Лакса [9].

ЗАмечАниЕ 3 . Если предположить, что в тензоре $P^{\prime}(4.1)$ коэффициенты $c_{k}$ являются не числами, а функциями от координат $q$, то решений уравнений $(2.5),(2.7)$ будет довольно много, но множество нетривиальных решений полной системы уравнений (2.14) останется тем же самым.

В уравнениях (2.13) можно использовать функцию Гамильтона натурального вида, определенную с точностью до точечных преобразований, т.е. в виде

$$
H=\widetilde{T}+V(q), \quad \widetilde{T}=\sum_{i, j=1}^{n} b_{i j}(q) p_{i} p_{j}+\sum_{k=1}^{n} b_{k}(q) p_{k}
$$

Здесь $b_{i j}(q)$ и $b_{k}(q)$ являются функциями от координат $q$ такими, что кинетическую энергию $\widetilde{T}$ можно привести к форме (2.1) точечными преобразованиями.

Используя эту возможность обобщения предложенного алгоритма, можно, например, получить некоторые системы Драша [19]. Мы ограничимся рассмотрением только одной из систем Драша, последней в его списке, для которой переменные 
разделения были не известны ранее и которая не является вырожденной системой Штеккеля, обладающей квадратичными интегралами движения [20].

ПРЕДЛОЖЕНИЕ 4. Одно из решений системы уравнений (2.18) на плоскости с псевдоевклидовой метрикой, т.е. при $H_{1}=p_{1} p_{2}+V\left(q_{1}, q_{2}\right)$, имеет вид

$$
H_{1}=p_{1} p_{2}-2 b \frac{2 a q_{1}-q_{2}}{\sqrt{q_{1}}}+\frac{C_{1}}{\sqrt{q_{1}}}+C_{2}\left(q_{2}-\frac{2 a}{3} q_{1}\right) .
$$

Подставляя соответствующий тензор Пуассона $P^{\prime}=P_{3}^{\prime}$ (4.3) при $\alpha=-1 / 2$ в оператор $N$ и вычисляя его собственные значения, мы получим переменные разделения для данной интегрируемой системь

$$
\lambda_{1,2}=-\frac{p_{2}}{4} \pm \frac{\sqrt{p_{2}^{2}+16 b \sqrt{q_{1}}}}{4}
$$

Второй интеграл движения $H_{2}$ является кубическим полиномом по импульсам.

ЗАмЕчАНИЕ 4. Другой возможностью расширить область применимости алгоритма является свобода выбора одного из интегралов $H_{1}, \ldots, H_{n}$, который можно отождествить с функцией Гамильтона натурального вида. В приведенных ранее примерах мы отождествляли $H_{1}=H$.

4.2. Квадратичный по импульсам тензор Пуассона. Рассмотрим следующий анзац для тензора Пуассона:

$$
P^{\prime}=\left(\begin{array}{cccc}
0 & h_{1}(q) p_{1}+h_{2}(q) p_{2} & \sum c_{i j}^{1} p_{i} p_{j}+\mathrm{g}_{1}(q) & \sum c_{i j}^{2} p_{i} p_{j}+\mathrm{g}_{2}(q) \\
* & 0 & \sum c_{i j}^{3} p_{i} p_{j}+\mathrm{g}_{3}(q) & \sum c_{i j}^{4} p_{i} p_{j}+\mathrm{g}_{4}(q) \\
* & * & 0 & f_{1}(q) p_{1}+f_{2}(q) p_{2} \\
* & * & * & 0
\end{array}\right)
$$

где $f_{i}, \mathrm{~g}_{i}, h_{i}$ - функции от координат $q$, а $c_{i j}^{k}$ - числовые параметры.

4.2.1. Квадратичные интегралы движения. Одно из решений уравнений (2.5), $(2.7),(2.13)$ с контрольной матрицей $F(2.20)$ имеет вид

$$
H_{1}=p_{1}^{2}+p_{2}^{2}+\frac{C_{3}}{2}\left(q_{1}^{2}+q_{2}^{2}\right)-\frac{C_{1}-C_{2}}{2\left(q_{1}+q_{2}\right)^{2}}-\frac{C_{1}+C_{2}}{2\left(q_{1}-q_{2}\right)^{2}}
$$

И

$$
P^{\prime}=\left(\begin{array}{cccc}
0 & p_{1} q_{2}-p_{2} q_{1} & -p_{1}^{2}+\mathrm{g}_{1}(q) & -p_{2} p_{1}+\mathrm{g}_{2}(q) \\
* & 0 & -p_{2} p_{1}+\mathrm{g}_{3}(q) & -p_{2}^{2}+\mathrm{g}_{4}(q) \\
* & * & 0 & p_{1} f_{1}(q)+p_{2} f_{2}(q) \\
* & * & * & 0
\end{array}\right)
$$

где

$$
\begin{gathered}
\mathrm{g}_{1}=\frac{q_{1}\left(C_{1} q_{1}^{3}+3 C_{2} q_{1}^{2} q_{2}+3 C_{1} q_{1} q_{2}^{2}+C_{2} q_{2}^{3}\right)}{\left(q_{1}-q_{2}\right)^{3}\left(q_{1}+q_{2}\right)^{3}}, \\
\mathrm{~g}_{2}=\frac{q_{1}\left(C_{2} q_{1}^{3}+3 C_{1} q_{1}^{2} q_{2}+3 C_{2} q_{1} q_{2}^{2}+C_{1} q_{2}^{3}\right)}{\left(q_{1}-q_{2}\right)^{3}\left(q_{1}+q_{2}\right)^{3}}, \\
\mathrm{~g}_{3}=q_{2} q_{1}^{-1} \mathrm{~g}_{1}(q), \quad \mathrm{g}_{4}=q_{2} q_{1}^{-1} \mathrm{~g}_{2}(q), \\
f_{1}=q_{1}^{-1} \mathrm{~g}_{2}(q), \quad f_{2}=q_{1}^{-1} \mathrm{~g}_{1}(q) .
\end{gathered}
$$


В этом случае входящий в уравнения (2.13) интеграл движения $H_{2}=\widetilde{H}_{2}-H_{1}^{2} / 4$ является комбинацией гамильтониана $H_{1}$ и независимого от него квадратичного полинома по импульсам $\widetilde{H}_{2}$ такого, что $\left\{H_{1}, \widetilde{H}_{2}\right\}=0$.

Подставляя тензор $P^{\prime}(4.8)$ в уравнения $(2.13)$ с контрольной матрицей $F$ вида (2.15), можно получить другое нетривиальное решение:

$$
H_{1}=p_{1}^{2}+p_{2}^{2}-\frac{C_{1}\left(q_{1}^{2}+q_{2}^{2}\right)}{\left(q_{1}+q_{2}\right)^{2}\left(q_{1}-q_{2}\right)^{2}}-\frac{2 C_{2} q_{1} q_{2}}{\left(q_{1}+q_{2}\right)^{2}\left(q_{1}-q_{2}\right)^{2}} .
$$

Как и ранее, второй интеграл движения $H_{2}$, входящий в уравнения $(2.13)$, является комбинацией двух квадратичных интегралов движения. Таким образом, мы опять получили интегрируемую систему с квадратичными интегралами движения, которая допускает разделение переменных в одной из криволинейных систем координат на плоскости.

4.2.2. Интегралы движения четвертого порядка по импульсам. Всюду далее мы не будем рассматривать интегрируемые системы, для которых второй интеграл движения $H_{2}$ в (2.13) является полиномом четвертой степени только формально, т.е. для которых $H_{2}$ является комбинацией гамильтониана $H_{1}$ и второго независимого квадратичного полинома $\widetilde{H}_{2}$. Также мы не будем рассматривать системы, которые допускают разделение переменных в исходных физических переменных $q_{1,2}$, т.е. системы, для которых $\partial^{2} V / \partial q_{1} \partial q_{2}=0$.

Итак, подставляя анзац (4.7) в уравнения (2.5), (2.7), мы получим, что функции $h_{i}$ либо постоянные $\left(h_{i}=\right.$ const $)$, либо линейные $\left(h_{i}=a_{i} q_{1}+b_{i} q_{2}\right)$. Рассмотрим эти два случая по отдельности.

ПреДлОЖЕнИЕ 5. Существуют всего два решения уравнений системъ (2.14) с контрольной матричей $F$ (2.15), гамильтонианом натурального вида $H_{1}$ и тензором Пуассона вида (4.7)

$$
P^{\prime}=\left(\begin{array}{cccc}
0 & 2 a p_{1}+2 b p_{2} & p_{1}^{2}+\mathrm{g}_{1}(q) & \mathrm{g}_{2}(q) \\
* & 0 & \mathrm{~g}_{3}(q) & p_{2}^{2}+\mathrm{g}_{4}(q) \\
* & * & 0 & f_{1}(q) p_{1}+f_{2}(q) p_{2} \\
* & * & * & 0
\end{array}\right)
$$

Если $a \neq 0$ и $b \neq 0$, то гамильтониан равен

$$
H_{1}=p_{1}^{2}+p_{2}^{2}+(a+b) C_{1} e^{\left(q_{2}-q_{1}\right) /(a+b)}-(a-b) C_{2} e^{\left(q_{2}+q_{1}\right) /(a-b)},
$$

при этом функиии, определяющие тензор Пуассона, имеют вид

$$
\begin{array}{lll}
f_{1}=C_{1} e^{\left(q_{2}-q 1\right) /(a+b)}-C_{2} e^{\left(q_{1}+q_{2}\right) /(a-b)}, & f_{2}=C_{1} e^{\left(q_{2}-q 1\right) /(a+b)}+C_{2} e^{\left(q_{1}+q_{2}\right) /(a-b)}, \\
\mathrm{g}_{1}=2 b f_{2}, \quad \mathrm{~g}_{2}=b f_{1}-a f_{2}, & \mathrm{~g}_{3}=-\mathrm{g}_{2}, \quad \mathrm{~g}_{4}=2 a f_{1} .
\end{array}
$$

Если либо $a=0$, либо $b=0$, то существует еще одно нетривиальное решение. При $a=0$ и $b=1$ гамильтониан имеет вид

$$
H_{1}=p_{1}^{2}+p_{2}^{2}+2 C_{1} e^{-q_{1}-q_{2}}+2 C_{2} e^{q_{2}-q_{1}}-\frac{C_{4} e^{2 q_{2}}}{2\left(C_{2} e^{2 q_{2}}-C_{1}\right)^{2}}-\frac{C_{5} e^{q_{2}}\left(C_{2} e^{2 q_{2}}+C_{1}\right)}{\left(C_{2} e^{2 q_{2}}-C_{1}\right)^{2}},
$$


а остальные функиии, входящие в тензор $P^{\prime}(4.9)$, равнь

$$
\begin{array}{ll}
f_{1}=\left(C_{2} e^{q_{2}}-C_{1} e^{-q_{2}}\right) e^{-q_{1}}, & f_{2}=\left(C_{2} e^{q_{2}}+C_{1} e^{-q_{2}}\right) e^{-q_{1}}, \\
\mathrm{~g}_{1}=2 f_{2}, \quad \mathrm{~g}_{2}=f_{1}+\frac{d \mathrm{~g}_{4}}{d q_{2}}, & \mathrm{~g}_{3}=f_{1}, \\
\mathrm{~g}_{4}=\frac{C_{4} e^{2 q_{2}}}{\left(C_{2} e^{2 q_{2}}-C_{1}\right)^{2}}+\frac{C_{5} e^{q_{2}}\left(C_{2} e^{2 q_{2}}+C_{1}\right)}{\left(C_{2} e^{2 q_{2}}-C_{1}\right)^{2}} &
\end{array}
$$

Интеграль движения $\mathrm{H}_{2}$ являются полиномами четвертой степени по импульсам.

Первое решение (4.10) отвечает открытой двухчастичной цепочке Тоды, связанной с корневой системой $\mathcal{D}_{2}$-типа.

Если $C_{4}=C_{5}=0$, то второе решение (4.11) также отвечает цепочке Тоды $\mathcal{D}_{2}$-типа. Однако если $C_{1}=0$ или $C_{2}=0$, то решение (4.11) отвечает открытой двухчастичной цепочке Тоды, связанной с корневой системой $\mathcal{B C}_{2}$-типа. В случае $C_{1}=C_{2}$ решение (4.11) отвечает деформации цепочки Тоды, предложенной Иноземцевым [17].

ПРЕДЛОЖЕНИЕ 6. Для уравнений (2.14) с контрольной матрицей $F$ (2.20) существуют только два явных нетривиальных решения вида (4.7) с $h_{i}=a_{i} q_{1}+b_{i} q_{2}$. Первое из них отвечает системе типа Холта с гамильтонианом

$$
H_{1}=p_{1}^{2}+p_{2}^{2}-\frac{C_{1}\left(2 q_{1}^{2}+9 q_{2}^{2}\right)}{6 q_{2}^{2 / 3}}+\frac{C_{2}}{q_{1}^{2}}+C_{3} q_{2}^{2 / 3}-\frac{C_{4}}{q_{2}^{2 / 3}}
$$

и тензором Пуассона

$$
P^{\prime}=\left(\begin{array}{cccc}
0 & 3 p_{1} q_{2} & p_{1}^{2}+C_{2} / q_{1}^{2} & p_{1} p_{2}+C_{1} q_{1} q_{2}^{1 / 3} \\
* & 0 & -3 C_{2} q_{2} / q_{1}^{3} & p_{1}^{2}-3 C_{1} q_{2}^{4 / 3}+C_{2} / q_{1}^{2}+2 C_{3} q_{2}^{2 / 3} \\
* & * & 0 & -C_{1} p_{1} q_{2}^{1 / 3}-C_{2} p_{2} / q_{1}^{3} \\
* & * & * & 0
\end{array}\right)
$$

Второе решение отвечает известной интегрируемой системе с потенииалом четвертой степени (биквадратичный потенииал)

$$
H_{1}=p_{1}^{2}+p_{2}^{2}-C_{1}\left(4 q_{1}^{4}+3 q_{1}^{2} q_{2}^{2}+\frac{q_{2}^{4}}{2}\right)+C_{2}\left(q_{1}^{2}+\frac{q_{2}^{2}}{4}\right)-\frac{C_{3}}{q_{1}^{2}}-\frac{C_{4}}{q_{2}^{2}}
$$

и тензором Пуассона

$$
P^{\prime}=\left(\begin{array}{cccc}
0 & p_{1} q_{2} & p_{1}^{2}+\mathrm{g}_{1}(q) & p_{1} p_{2}+\mathrm{g}_{2}(q) \\
* & 0 & \mathrm{~g}_{3}(q) & p_{1}^{2}+2 p_{2}^{2}+\mathrm{g}_{4}(q) \\
* & * & 0 & p_{1} f_{1}(q)+p_{2} f_{2}(q) \\
* & * & * & 0
\end{array}\right)
$$


где

$$
\begin{array}{lr}
f_{1}=-C_{1} q_{2}^{3}, & f_{2}=2 C_{1} q_{1}\left(4 q_{1}^{2}+3 q_{2}^{2}\right)-C_{2} q_{1}-\frac{C_{3}}{q_{1}^{3}}, \\
\mathrm{~g}_{1}=-4 C_{1} q_{1}^{4}+C_{2} q_{1}^{2}-\frac{C_{3}}{q_{1}^{2}}, & \mathrm{~g}_{2}=C_{1} q_{1} q_{2}^{3}, \quad \mathrm{~g}_{3}=4 \mathrm{~g}_{2}+q_{2} f_{2}, \\
\mathrm{~g}_{4}=-C_{1}\left(4 q_{1}^{4}+6 q_{1}^{2} q_{2}^{2}+q_{2}^{4}\right)+C_{2}\left(q_{1}^{2}+\frac{q_{2}^{2}}{2}\right)-\frac{C_{3}}{q_{1}^{2}} .
\end{array}
$$

Соответствующие вторые интеграль движения $\mathrm{H}_{2}$ совпадают с известными полиномами четвертой степени по импульсам [2], [9].

Во втором случае можно сделать контракцию переменных специального вида [21] и получить систему типа Энона-Эйлеса с потенциалом третьей степени

$$
H_{1}=p_{1}^{2}+p_{2}^{2}+\frac{1}{3} q_{1}\left(16 q_{1}^{2}+3 q_{2}^{2}\right)+\frac{C_{1}}{q_{2}^{2}} .
$$

Выпишем соответствующий тензор Пуассона явно:

$$
P^{\prime}=\left(\begin{array}{cccc}
0 & q_{2} p_{1} & p_{1}^{2}+16 q_{1}^{3} / 3 & p_{1} p_{2}-q_{2}^{3} / 6 \\
* & 0 & 8 q_{2} q_{1}^{2}+q_{2}^{3} / 3 & p_{1}^{2}+2 p_{2}^{2}+2 q_{1} q_{2}^{2}+16 q_{1}^{3} / 3 \\
* & * & 0 & -p_{2}\left(8 q_{1}^{2}+q_{2}^{2}\right) \\
* & * & * & 0
\end{array}\right)
$$

ЗАмечание 5. Собственные значения операторов $N$, построенных с помощью тензоров (4.15) и (4.17), удовлетворяют тривиальным уравнениям движения $\dot{\lambda}_{1,2}=0$ и, следовательно, не являются переменными разделения для системы с потенциалом четвертой степени (4.14) и системы типа Энона-Эйлеса (4.16).

Кроме этих решений уравнений $(2.5),(2.7)$, (2.13) существуют еще несколько решений вида (4.7), для которых функции Гамильтона не могут быть выписаны явно. Во всех этих случаях функции $f_{i}(q), \mathrm{g}_{i}(q)$ и полином четвертого порядка по импульсам $H_{2}(p, q)$ выражаются через потенциал $V\left(q_{1}, q_{2}\right)$, который, в свою очередь, удовлетворяет различным дифференциальным уравнениям второго и третьего порядка в частных производных. В качестве примера мы выпишем одно из полученных уравнений второго порядка:

$$
\left(q_{1} q_{2}+C_{1}\right)\left(\frac{\partial^{2}}{\partial q_{1}^{2}}-\frac{\partial^{2}}{\partial q_{2}^{2}}\right) V+3\left(q_{2} \frac{\partial}{\partial q_{1}}-q_{1} \frac{\partial}{\partial q_{2}}\right) V=\left(q_{1}^{2}-q_{2}^{2}+C_{2}\right) \frac{\partial^{2}}{\partial q_{1} \partial q_{2}} V .
$$

Подобные уравнения второго порядка рассматривались, например, в работах [2], [22]. Уравнения третьего порядка в частных производных, которые используются для описания интегрируемых систем на плоскости, практически не встречаются в литературе. Эти системы требуют отдельного детального исследования.

Как и ранее, в уравнениях (2.13) можно использовать функцию Гамильтона натурального вида (4.6), определенную с точностью до канонических точечных преобразований.

2 Теоретическая и математическая физика, т. 149, № 2, 2006 г. 
ПрЕДЛОЖЕНИЕ 7. В случае псевдоевклидовой метрики на плоскости существуют два явных решения уравнений (2.14) вида (4.7). В первом случае

$$
\begin{gathered}
H_{1}=p_{1} p_{2}+C_{1}\left(4 q_{1}^{2}+5 \alpha q_{1} q_{2}+\alpha^{2} q_{2}^{2}\right)+\frac{C_{2}\left(q_{1}+\alpha q_{2}\right)}{q_{2}^{1 / 3}}+\frac{C_{3}}{q_{2}^{2 / 3}}+\frac{C_{4}}{q_{2}^{4 / 3}}, \\
P^{\prime}=\left(\begin{array}{cccc}
0 & 3 q_{2} p_{1} & p_{1}^{2}-6 C_{1} q_{2}\left(q_{1}+\alpha q_{2}\right) & \alpha p_{1}^{2}-3 p_{1} p_{2}-3 C_{1}\left(q_{1}+\alpha q_{2}\right)\left(2 q_{1}+\alpha q_{2}\right)-\frac{C_{2}\left(q_{1}+\alpha q_{2}\right)}{q_{2}^{1 / 3}}+\frac{2 C_{4}}{q_{2}^{4 / 3}} \\
* & 0 & 18 C_{1} q_{2}^{2} & 9 C_{1} q_{2}\left(2 q_{1}+\alpha q_{2}\right)+3 C_{2} q_{2}^{2 / 3} \\
* & * & 0 & \left(3 C_{1}\left(2 q_{1}-\alpha q_{2}\right)+\frac{C_{2}}{q_{2}^{1 / 3}}\right) p_{1}+18 C_{1} q_{2} p_{2} \\
* & * & * & 0
\end{array}\right),
\end{gathered}
$$

во втором случае

$$
\begin{gathered}
H_{1}=p_{1} p_{2}+\left(C_{1}+\frac{C_{2}}{\sqrt{q_{2}}}\right) q_{1}+C_{3} q_{2}+\frac{3 C_{2} C_{3}}{C_{1}} \sqrt{q_{2}}, \\
P^{\prime}=\left(\begin{array}{cccc}
0 & 4 q_{2} p_{1} & p_{1}^{2}+q_{1}+\alpha q_{2} & \left(6 C_{1}+4 \alpha\right) p_{1}^{2}-2 p_{1} p_{2}+2\left(C_{1}+\alpha\right) q_{1}+2 C_{1} \alpha\left(\left(\sqrt{q_{2}}+\frac{C_{2}}{C_{1}}\right)^{2}-\frac{C_{2}^{2}\left(6 C_{1}+\alpha\right)}{4 C_{1}^{3}}\right) \\
* & 0 & 2 q_{2} & p_{1}^{2}+q_{1}+\left(\alpha+4 C_{1}\right) q_{2}+4 C_{2} \sqrt{q_{2}} \\
* & * & 0 & -\frac{C_{3}}{C_{1}} p_{1}+p_{2} \\
* & * & * & 0
\end{array}\right),
\end{gathered}
$$

где $\alpha=\left(C_{3}-2 C_{1}^{2}\right) / C_{1}$.

Эти интегрируемые системы с интегралами движения $H_{2}$ четвертой степени по импульсам являются новыми интегрируемыми системами.

4.3. Нормированные тензоры Пуассона. В данном разделе мы рассмотрим нормированные тензоры Пуассона

$$
P^{\prime}=\operatorname{det}\left(\widehat{P}^{\prime}\right)^{-1} \widehat{P}^{\prime}
$$

где $\widehat{P}^{\prime}$ - кососимметричный тензор вида (4.7).

ПреДлОЖЕНИЕ 8. Существуют толъко два нетривиалъных решения уравнений (2.14) с матричей $F(2.20)$ и нормированным тензором Пуассона $P^{\prime}$ (4.18), в котором

$$
\widehat{P}^{\prime}=\left(\begin{array}{cccc}
0 & p_{1} q_{2} & -p_{2}^{2}+\mathrm{g}_{1}(q) & p_{1} p_{2}+\mathrm{g}_{2}(q) \\
* & 0 & \mathrm{~g}_{3}(q) & p_{2}^{2}+\mathrm{g}_{4}(q) \\
* & * & 0 & f_{1}(q) p_{1}+f_{2}(q) p_{2} \\
* & * & * & 0
\end{array}\right)
$$

Первое решение отвечает системе типа Энона-Эйлеса с гамильтонианом

$$
H_{1}=p_{1}^{2}+p_{2}^{2}-C_{1} q_{1}\left(16 q_{1}^{2}+3 q_{2}^{2}\right)-2 C_{2} q_{1} .
$$

В этом случае функции, входящие в (4.19), равнь

$$
\begin{array}{ll}
f_{1}=0, & f_{2}=3 C_{1}\left(8 q_{1}^{2}+q_{2}^{2}\right)+C_{2}, \\
\mathrm{~g}_{1}=3 C_{1} q_{1} q_{2}^{2}, & \mathrm{~g}_{2}=\frac{C_{1}}{2} q_{2}^{3}, \\
\mathrm{~g}_{3}=4 \mathrm{~g}_{2}-f_{2} q_{2}, & \mathrm{~g}_{4}=-\mathrm{g}_{1} .
\end{array}
$$


Второе решение отвечает системе с потенииалом четвертой степени

$$
H_{1}=p_{1}^{2}+p_{2}^{2}+C_{1}\left(8 q_{1}^{4}+6 q_{1}^{2} q_{2}^{2}+q_{2}^{4}\right)+C_{2}\left(4 q_{1}^{2}+q_{2}^{2}\right)+\frac{C_{3}}{q_{1}^{2}} .
$$

В этом случае функиии, входящие в (4.19), имеют вид

$$
\begin{array}{ll}
f_{1}=2 C_{1} q_{2}^{3}, & f_{2}=-4 C_{1} q_{1}\left(3 q_{2}^{2}+4 q_{1}^{2}\right)-4 C_{2} q_{1}+\frac{C_{3}}{q_{1}^{3}}, \\
\mathrm{~g}_{1}=-\left(C_{1}\left(q_{2}^{2}+6 q_{1}^{2}\right)+C_{2}\right) q_{2}^{2}, & \mathrm{~g}_{2}=-2 C_{1} q_{1} q_{2}^{3}, \\
\mathrm{~g}_{3}=4 \mathrm{~g}_{2}-f_{2} q_{2}, & \mathrm{~g}_{4}=-\mathrm{g}_{1} .
\end{array}
$$

Соответствующие вторые интегралы движения, которые являются решениями уравнений (2.13), имеют вид

$$
H_{2}=\operatorname{det}\left(\widehat{P}^{\prime}\right)=\sqrt{p_{2}^{4}+q_{1} f_{1} p_{1}^{2}+\left(q_{2} f_{2}+\mathrm{g}_{3}\right) p_{1} p_{2}-2 \mathrm{~g}_{1} p_{2}^{2}-\mathrm{g}_{1}^{2}+\mathrm{g}_{2} \mathrm{~g}_{3}} .
$$

Квадраты этих интегралов движения являются полиномами четвертой степени по импульсам, построенными ранее (см. [2], [9]).

В обоих случаях соответствующие собственные значения оператора $N$ тривиальны: $\lambda_{1,2}= \pm 1$. Переменные разделения или переменные Дарбу-Нийенхейса $(x, y)$ определяются не собственными значениями оператора $N$, а с помощью канонических преобразований $z=(q, p) \rightarrow \zeta=(x, y)$, которые диагонализуют оператор $N(2.10)$.

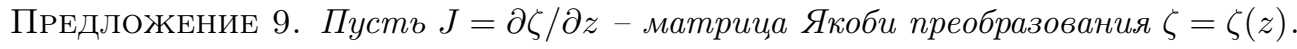
Решения системь уравнений

$$
J P J^{\mathrm{T}}=P, \quad J N J^{-1}=\operatorname{diag}(1,-1,1,-1)
$$

относительно $\zeta(p, q)$ являются переменными разделения для системы Энона-Эйлеса и системы с потенииалом четвертой степени, построенными ранее в рабоme [21].

Первое уравнение системы (4.20) означает, что искомое преобразование координат $z \rightarrow \zeta$ является каноническим преобразованием. Второе уравнение в (4.20) означает, что данное преобразование приводит оператор $N$ к диагональному виду. Уравнения (4.20) решались с помощью системы символьных вычислений Maple.

Взаимосвязь таких канонических преобразований, диагонализующих оператор $N$, и преобразований Беклунда, приводящих к разделению переменных, более подробно будет рассмотрена в последующих публикациях.

\section{5. ЗАКЛЮЧЕНИЕ}

В данной работе предложен новый прямой метод построения интегрируемых систем натурального вида. В этом методе интегралы движения являются решениями переопределенной системы алгебраических и дифференциальных уравнений 
в частных производных, полученной из условия совместности тензоров Пуассона $(2.5),(2.7)$ и условия инвариантности билагранжева распределения относительно действия оператора рекурсии (2.13).

Достоинством предлагаемого метода является достаточно легкая его реализация с помощью любой системы символьных вычислений. Одна из возможных реализаций приведена в приложении. Тем самым задача о построении и классификации бигамильтоновых интегрируемых систем достаточно широкого класса сведена к простым компьютерным вычислениям.

K недостаткам предложенного алгоритма относятся необходимость выбора анзаца для тензора Пуассона и использование контрольных матриц $F$ специального вида. Основной проблемой при этом является необходимость такого выбора, чтобы множество соответствующих интегрируемых систем было, с одной стороны, достаточно узким, для того чтобы найти все эти системы в расчетах на компьютере, а с другой стороны, это множество должно быть достаточно широким и включать в себя нетривиальные интегрируемые системы.

В качестве примера мы использовали очень простые анзацы (4.1) и (4.7) и, тем не менее, воспроизвели большинство из известных интегрируемых систем на плоскости с интегралами третьей и четвертой степени по импульсам.

Вторым недостатком алгоритма является необходимость решения сильно переопределенной системы уравнений, так что при использовании стандартных процедур вычислений время, необходимое для расчетов, растет экспоненциально как с ростом размерности $n=\operatorname{rank} P^{\prime}$, так и с увеличением степени матричного полинома $P^{\prime}$.

\section{ПРИЛОЖЕНИЕ}

\section{Программа для построения бигамильтоновых систем}

Данная работа, так же как и работы [11], может быть отнесена к активно развивающемуся разделу вычислительной математической физики. Поэтому далее приведена программная реализация описанного выше алгоритма построения бигамильтоновых систем, выполненная в среде символьных вычислений Maple. Строки программы помечены символом >, остальное представляет собой краткий комментарий.

Итак, загрузим необходимую нам библиотеку встроенных процедур, зададим размерность фазового пространства и определим канонические переменные:

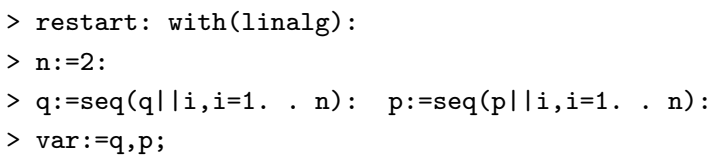

Теперь введем канонический тензор Пуассона:

$>$ ed:=array(identity, 1. . n,1. . n): $\mathrm{z}:=\operatorname{array}(\operatorname{sparse}, 1 . \mathrm{n}, 1 . \cdot \mathrm{n})$ :

$>$ P1:=blockmatrix $(2,2,[z, e d,-e d, z])$;

и анзац для второго тензора Пуассона

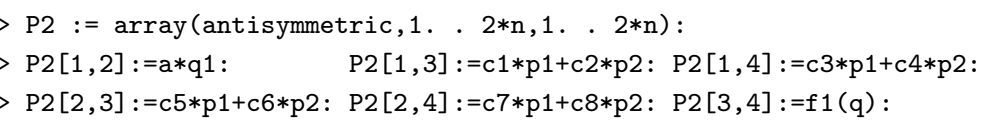


в который входят неопределенные коэффициенты $c_{1}, \ldots, c_{8}$ и функция $f_{1}(q)$.

Определим скобки Пуассона, отвечающие тензорам $P^{\prime}$ и $P+\lambda P^{\prime}$ :

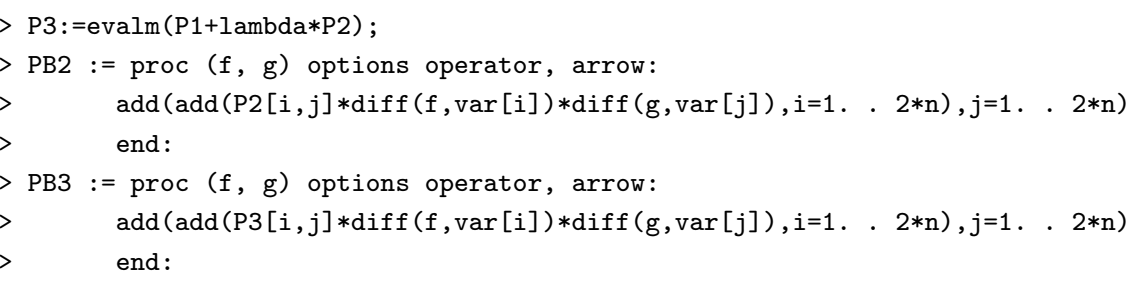

После этого выпишем тождества Якоби (2.6):

$>\mathrm{f}:=\mathrm{F}(\mathrm{var}) ; \mathrm{g}:=\mathrm{G}(\mathrm{var}): \mathrm{h}:=\mathrm{H}(\mathrm{var}):$

$>$ Y2: =simplify (PB2 (PB2 $(f, g), h)+P B 2(P B 2(g, h), f)+P B 2(P B 2(h, f), g)):$

$>\mathrm{Y} 3:=\operatorname{simplify}(\mathrm{PB} 3(\mathrm{~PB} 3(\mathrm{f}, \mathrm{g}), \mathrm{h})+\mathrm{PB} 3(\mathrm{~PB} 3(\mathrm{~g}, \mathrm{~h}), \mathrm{f})+\mathrm{PB} 3(\mathrm{~PB} 3(\mathrm{~h}, \mathrm{f}), \mathrm{g}))$ :

и создадим систему уравнений $(2.5),(2.7)$

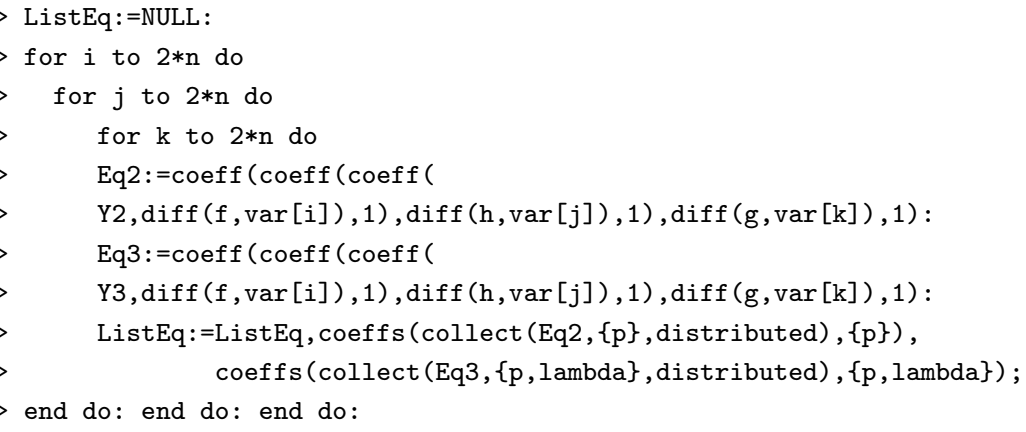

Далее построим оператор $N^{*}$ и его минимальный характеристический полином $\Delta(\lambda)$ :

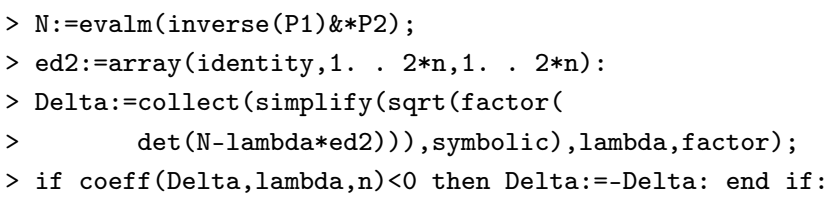

Теперь у нас все готово для определения контрольной матрицы $F(2.20)$ :

sigma: =vector $(n)$ :

for $\mathrm{k}$ to $\mathrm{n}$ do sigma[k]:=--coeff (Delta,lambda,n-k); end do:

$\mathrm{F}:=\operatorname{delcols}$ (augment (sigma, ed), $\mathrm{n}+1 . \mathrm{n}+1)$ :

Введем функцию Гамильтона натурального вида $H=H_{1}$ и остальные интегралы движения $H_{k}(p, q)$ в виде неопределенных пока функций и вычислим соответствующие им дифференциалы:

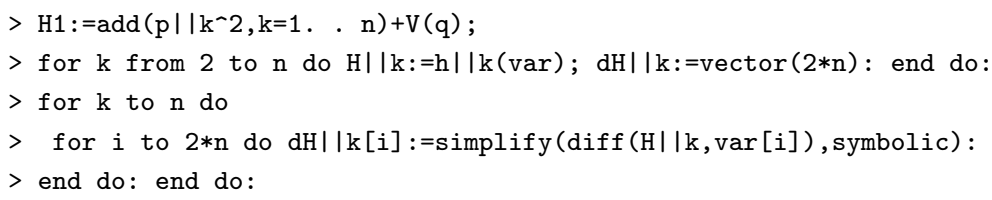

Нам осталось задать уравнения (2.13):

$>$ ListEqH: $=$ NULL :

$>$ for $i$ to $n$ do 
$>\quad Z|| i:=\operatorname{simplify}(\operatorname{evalm}(N \& * d H|| i-\operatorname{add}(F[i, j] * d H|| j, j=1 . \quad . n)))$ :

$>$ ListEqH: $=$ ListEqH, seq $(\mathrm{Z}|| \mathrm{i}[\mathrm{k}], \mathrm{k}=1.2 * \mathrm{n})$ :

$>$ end do:

и решить полученную полную систему уравнений

$>$ Ans: $=\operatorname{pdsolve}(\{$ ListEq, ListEqH $\},\{f 1(q), V(q), \operatorname{seq}(H|| k, k=2 . \quad n)\}$,

$>\quad \operatorname{parameters}=\{\operatorname{seq}(c|| j, j=1 . .8)\})$;

С помощью данной программы построение интегрируемых систем из п. 4.1 занимает несколько секунд на обычном персональном компьютере, а построение интегрируемых систем из п. 4.2 и 4.3 занимает несколько минут.

Если мы хотим исключить тривиальные решения, а это лучше всего сделать для уменьшения времени работы, то к построенным выше уравнениям необходимо добавить дополнительные уравнения, например $\partial H_{k} / \partial p_{i} \neq 0$ и $\partial^{2} V\left(q_{1}, q_{2}\right) / \partial q_{1} \partial q_{2} \neq 0$.

Благодарности. Автору приятно поблагодарить И. В. Комарова за многочисленные полезные обсуждения и интерес к работе. Работа выполнена при финансовой поддержке РФФИ (грант № 06-01-00140).

\section{Список литературы}

[1] F. Magri, "Eight lectures on integrable systems", Integrability of Nonlinear Systems (Proc. of the CIMPA school (Pondicherry Univ., India, January 8-25, 1996)), Lect. Notes Phys., 495, eds. Y. Kosmann-Schwarzbach et al., Springer, Berlin-Heidelberg, 1997, 256-296.

[2] J. Hietarinta, Phys. Rep., 147 (1987), 87-154.

[3] G. Falqui, F. Magri, M. Pedroni, J. Nonlinear Math. Phys., 8 (2001), 118-127.

[4] G. Falqui, M. Pedroni, Math. Phys. Anal. Geom., 6 (2003), 139-179.

[5] Y. Kosmann-Schwarzbach, F. Magri, Ann. Inst. H. Poincaré, Phys. Théor., 53 (1990), 35-81.

[6] B. Fuchssteiner, A. Fokas, Physica D, 4 (1981), 47-66.

[7] И. М. Гельфанд, И. Я. Дорфман, Функи. анализ и его прилож., 13:4 (1979), 13-30.

[8] R. Brouzet, J. Math. Phys., 34 (1993), 1309-1313.

[9] А. М. Переломов, Интегрируемъе системъ классической механики и алгебры Ли, Наука, М., 1990.

[10] C. Bartocci, G. Falqui, M. Pedroni, Differ. Geom. Appl., 21:3 (2004), 349-360.

[11] A. V. Tsiganov, Yu. A. Grigoryev, Regul. Chaotic Dyn., 10 (2005), 413-422; А. В. Цыганов, Ю. А. Григорьев, Нелинейная механика, 1 (2005), 163-179.

[12] A. Ibort, F. Magri, G. Marmo, J. Geom. Phys., 33 (2000), 210-228.

[13] A. V. Tsiganov, J. Math. Phys., 40 (1999), 279-298.

[14] S. Benenti, J. Math. Phys., 38 (1997), 6578-6602.

[15] P. A. Damianou, Rev. Math. Phys., 16 (2004), 175-241.

[16] R. L. Fernandes, J. Phys. A, 26 (1993), 3797-3803.

[17] V.I. Inozemtsev, Commun. Math. Phys., 121 (1989), 629-643.

[18] C. R. Holt, J. Math. Phys., 23 (1982), 1037-1046.

[19] J. Drach, C. R. Acad. Sci. Paris, 200 (1935), 22-26.

[20] А. В. Цыганов, ТMФ, 124:3 (2000), 426-444.

[21] S. Rauch-Wojciechowski, A. V. Tsiganov, J. Phys. A, 29 (1996), 7769-7778.

[22] M. Wojciechowska, S. Wojciechowski, Phys. Lett. A, 105 (1984), 11-14.

Поступила в редакцию 21.03.2006, после доработки 14.06.2006 